Research Paper

\title{
Comparison of FIB-4 index and aspartate aminotransferase to platelet ratio index on carcinogenesis in chronic hepatitis B treated with entecavir
}

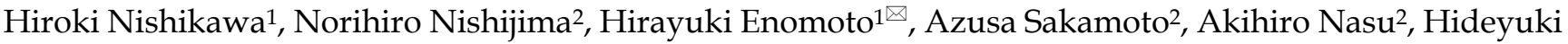 \\ Komekado ${ }^{2}$, Takashi Nishimura ${ }^{1}$, Ryuichi Kita ${ }^{2}$, Toru Kimura ${ }^{2}$, Hiroko Iijima ${ }^{1}$, Shuhei Nishiguchi ${ }^{1}$, Yukio \\ Osaki $^{2}$ \\ 1. Division of Hepatobiliary and Pancreatic disease, Department of Internal Medicine, Hyogo College of Medicine, Hyogo, Japan; \\ 2. Department of Gastroenterology and Hepatology, Osaka Red Cross Hospital, Osaka, Japan. \\ $\square$ Corresponding author: Hirayuki Enomoto, MD, PhD, Department of Hepatobiliary and Pancreatic disease, Department of Internal Medicine, Hyogo College \\ of Medicine, Hyogo, Japan. 1-1, Mukogawacho, Nishinomiyashi, Hyogo, 663-8501, Japan. Tel: +81-798-45-6111; Fax: +81-798-45-6608 E-mail: \\ enomoto@hyo-med.ac.jp. \\ (1) Ivyspring International Publisher. This is an open access article distributed under the terms of the Creative Commons Attribution (CC BY-NC) license \\ (https:// creativecommons.org/licenses/by-nc/4.0/). See http://ivyspring.com/terms for full terms and conditions.
}

Received: 2016.06.18; Accepted: 2016.09.19; Published: 2017.01.11

\begin{abstract}
Aims: We sought to compare the effects of FIB-4 index and aspartate aminotransferase to platelet ratio index (APRI) on hepatocellular carcinoma $(\mathrm{HCC})$ incidence in chronic hepatitis $B(\mathrm{CHB})$ patients undergoing entecavir (ETV) therapy.

Patient and methods: A total of 338 nucleosides analogue therapy naive $\mathrm{CHB}$ patients initially treated with ETV were analyzed. The optimal cutoff points in each continuous variable were determined by receiver operating curve (ROC) analysis. The effects of FIB-4 index and APRI on $\mathrm{HCC}$ incidence were compared using time-dependent ROC analysis and factors linked to HCC incidence were also examined using univariate and multivariate analyses.

Results: There were 215 males and 123 females with the median age of 52 years and the median baseline HBV-DNA level of 6.6 log copies/ml. The median follow-up interval after the initiation of ETV therapy was 4.99 years. During the follow-up period, 33 patients $(9.8 \%)$ developed HCC. The 3-, 5- 7-year cumulative HCC incidence rates in all cases were $4.4 \%, 9.2 \%$ and $13.5 \%$, respectively. In the multivariate analysis, FIB-4 index revealed to be an independent predictor associated with HCC incidence, while APRI was not. In the time-dependent ROC analyses for all cases and for all subgroups analyses stratified by viral status or cirrhosis status, all area under the ROCs in each time point (2-, 3-, 4-, 5-, 6-, and 7-year) of FIB-4 index were higher than those of APRI.

Conclusion: FIB-4 index rather than APRI can be a useful predictor associated with HCC development for CHB patients undergoing ETV therapy.
\end{abstract}

Key words: Chronic hepatitis B, Entecavir, Carcinogenesis, FIB-4 index, APRI.

\section{Introduction}

Hepatocellular carcinoma (HCC) is one of leading etiologies of cancer related death worldwide. $[1,2,3]$ Chronic hepatitis B (CHB) infection is one of leading causes of HCC incidence particularly in Asian countries such as China, Korea and Taiwan, which accounts for approximately $50-60 \%$ of HCC cases in the world. [1, 2, 4, 5] A current systematic review estimated that in 2010, about 248 million individuals had HB surface antigen (HBsAg) positive status globally. [6] The ultimate goal for CHB therapy is to 
suppress liver related complications including liver decompensation and liver carcinogenesis, which together cause more than 500000 deaths annually. [4, 7-11] Previous investigations demonstrated that older age, higher HBV-DNA viral load, higher alanine aminotransferase (ALT) level, lower serum albumin level, higher total bilirubin level, presence of liver cirrhosis (LC), lower platelet count, core promoter mutations and $\mathrm{HBe}$ antigen positivity were associated with HCC incidence in patients with CHB. [7, 10, 12-17] Furthermore, multi-centric liver carcinogenesis is closely related to liver fibrosis and persistent liver inflammation. [18, 19]

The American Association for Study of Liver Disease (AASLD) guidelines have evolved over time along with the approval of new antiviral agents or introduction of novel clinical evidence. In the current AASLD guidelines, either entecavir (ETV) or tenofovir disoproxil fumarate are recommended to use as the first-line nucleoside analogues (NAs) in patients with $\mathrm{CHB}$ due to their potent viral suppression with a lower risk of drug resistance and excellent preventive effect for HCC development. [11, $12,20-24]$

FIB-4 index and aspartate aminotransferase (AST) to platelet ratio index (APRI), which are simple and noninvasive liver fibrosis markers, were originally developed for predicting the degree of liver fibrosis in patients with chronic hepatitis C. Their usefulness in patients with $\mathrm{CHB}$ have also been verified. A recent meta-analysis for the usefulness of FIB-4 index and APRI in CHB indicated that FIB-4 index and APRI can identify CHB-related fibrosis with a moderate sensitivity and accuracy. [25] Another meta-analysis of FIB-4 index in CHB presented that FIB-4 index is helpful for predicting significant fibrosis and LC in CHB patients. [26] Thus, these biomarkers can provide useful clinical information of CHB-related liver fibrosis for clinicians. On the other hand, in terms of clinical efficacy of FIB-4 index and APRI on CHB-related liver carcinogenesis, a Korean study reported that high FIB-4 index was a highly predictive factor for carcinogenesis in 986 Korean HBsAg carriers aged 40 years or more. [27] In addition, Hann, et al. demonstrated that APRI might be a predictor of HCC development in $\mathrm{CHB}$ patients irrespective of the presence or absence of LC. [28] However, to the best of our knowledge, the effects of FIB-4 index and APRI on liver carcinogenesis in CHB patients receiving ETV therapy have not been fully examined. As described above, since ETV is a first line NA for the treatment of $\mathrm{CHB}$, clarifying these issues are essential problems.
The aims of the current study were therefore to compare the effects of FIB-4 index and APRI on liver carcionogenesis in $\mathrm{CHB}$ patients undergoing ETV therapy.

\section{Patients and methods}

\section{Patients}

From April 2006 until February 2015, a total of $363 \mathrm{CHB}$ patients who initially received ETV therapy and no apparent evidence of HCC on imaging findings were admitted to the Department of Gastroenterology and Hepatology, Osaka Red Cross Hospital, Osaka, Japan and the Division of Hepatobiliary and Pancreatic disease, Department of Internal Medicine, Hyogo College of Medicine, Hyogo, Japan. All are NA treatment naïve and they had detectable HBs antigen for more than six months. In addition, all subjects had no clinical evidence of detectable hepatitis $C$ virus antibody, and no apparent evidence of alcoholic abuse or drug-induced liver injury. In baseline data in analyzed subjects, we adopted data at the start of ETV treatment. All patients were initially treated by ETV with a daily dose of $0.5 \mathrm{mg}$. ETV therapy for $\mathrm{CHB}$ was chosen based on the decision of each attending physician. Of these patients, subjects with HCC development within one year after the start of ETV therapy $(n=14)$, those with observation period after the start of ETV therapy less than one year $(n=4)$ and insufficient baseline clinical data $(n=7)$ were excluded from the current analysis. Thus, the present study cohort was comprised of a total of $338 \mathrm{CHB}$ patients. All analyzed cases were treated by ETV for at least one year. LC was diagnosed pathologically and/or radiologically. In cases without liver biopsy, LC was diagnosed through findings of imaging studies: clinical characteristics indicative of portal hypertension such as varices, ascites or splenomegaly and a shrunken, deformed and nodular liver as noted on liver imaging. The main outcome measure in our study is development of HCC. HCC was diagnosed based on Japanese guidelines. For subjects with atypical imaging findings, ultrasound-guided tumor biopsy was performed for histological evaluation. Based on the previous reports, FIB-4 index was calculated as follows: age (years) $\times$ AST $(\mathrm{IU} / \mathrm{l}) /$ platelet count $\left.\left(\times 10^{9} / 1\right) \times \sqrt{\text { ALT }}(\mathrm{IU} / \mathrm{l})\right)$ and APRI score was calculated as follows: (AST/upper limit of normal)/platelet count $\left(\right.$ expressed as platelets $\left.\times 10^{9} / 1\right) \times 100$. [29, 30] The effects of FIB-4 index and APRI on HCC incidence were compared using time-dependent receiver operating characteristics (ROC) analysis (described later) and factors linked to HCC incidence were examined using univariate and multivariate analyses. 
This study was approved by ethics committees in each hospital and the present study protocol strictly adhered to all of the provisions of the Declaration of Helsinki.

\section{Serological studies of HBV markers}

Blood testing for HBs antigen and HBe antigen and $\mathrm{HBe}$ antibody were performed using commercially available kits (Abbott or Fujirebio, Tokyo, Japan). We measured HBV-DNA load by using the COBAS Amplicor HBV Monitor Test (Roche Diagnostics, Tokyo, Japan: linear range of detection; 2.6-7.6 log copies $/ \mathrm{ml}$ ) or by using the COBAS Taqman HBV Test v2.0 (Roche Diagnostics, Tokyo, Japan: linear range of detection; 2.1-9.0 log copies $/ \mathrm{ml}$ ).

\section{HCC surveillance and follow-up}

Observation after the start of ETV therapy consisted of radiological assessment by using ultrasonography, computed tomography and/or magnetic resonance imaging for HCC incidence every 2 to 6 months and periodical blood tests such as HBV-related laboratory markers and tumor markers. In patients with HCC incidence, most adequate therapy for each case was chosen primarily based on Japanese guidelines. [31-33]

\section{Statistical analysis}

In continuous variables, we performed ROC curve analysis of HCC incidence for selection of the optimal cutoff point that maximized the sum of specificity and sensitivity and we classified them into two groups using these cutoff points and treated them as nominal covariates. When selecting optimal cutoff points of continuous parameters, we took median follow-up period for the entire study cohort into consideration because HCC development is time-dependent. Kaplan-Meier curves for HCC development were generated and compared by using the log-rank test. Variables with $P<0.05$ in the univariate analysis were incorporated into the multivariate analyses. Furthermore, we analyzed time-dependent ROC curves of FIB-4 index and APRI for HCC incidence and compared between area under the ROCs (AUROCs) for FIB-4 index and APRI in each time point (2-, 3-, 4-, 5-, 6-, and 7-year). [34]

Time interval for HCC incidence was defined as duration from the date of the initiation of ETV therapy until the date of first confirmed HCC on imaging findings. In patients without HCC development, observation period was calculated as the time from the date of the initiation of ETV therapy to the last follow-up date. Data are presented as median (range) unless otherwise stated. We treated variables with $P<0.05$ as statistically significant variables. Statistical analysis was performed by using the JMP 11 (SAS Institute Inc., Cary, NC).

\section{Results}

\section{Baseline characteristics}

The baseline characteristics for all subjects $(n=338)$ in this study are presented in table 1 .

There were 215 males and 123 females with the median age of 52 years (range, 18-82 years) and the median baseline HBV-DNA level of $6.6 \mathrm{log}$ copies $/ \mathrm{ml}$ (range, 2.1-9.0 log copies/ml). Ninety-one patients $(26.9 \%)$ had LC on radiologic findings, while 163 patients $(48.2 \%)$ had HBe antigen positivity. The median (range) follow-up interval after the initiation of ETV therapy was 4.99 years (1.03-9.98 years). In terms of viral response to ETV therapy, 239 patients $(70.7 \%)$ had HBV-DNA level $<2.6 \log$ copies $/ \mathrm{ml}$ at six months from the start of ETV therapy. In this study, FIB-4 index ranged from 0.233 to 55.528 (median, 1.983), while APRI ranged from 0.061 to 28.977 (median, 0.900).

Table 1. Baseline characteristics.

\begin{tabular}{ll}
\hline Variables & $\mathrm{N}=338$ \\
\hline Age (years) & $52(18-82)$ \\
Gender, male / female & $215 / 123$ \\
AST $(\mathrm{IU} / \mathrm{l})$ & $46(7-1395)$ \\
ALT (IU/l) & $58(7-1450)$ \\
Serum albumin $(\mathrm{g} / \mathrm{dl})$ & $4.1(2.0-5.1)$ \\
Total bilirubin $(\mathrm{mg} / \mathrm{dl})$ & $0.8(0.2-11.1)$ \\
Platelet count $\left(\times 10^{4} / \mathrm{mm}^{3}\right)$ & $17.15(1.6-41.7)$ \\
Pretreatment alfa-fetoprotein $(\mathrm{ng} / \mathrm{ml})$ & $5.5(0.9-1039.5)$ \\
Presence of liver cirrhosis, yes / no & $91 / 247$ \\
HBV-DNA (Log copies/ml) & $6.6(2.1-9.0)$ \\
HBe antigen positivity, yes / no & $163 / 175$ \\
\hline
\end{tabular}

Data are expressed as number or median value (range). AST; aspartate aminotransferase, ALT; alanine aminotransferase, HBV; hepatitis B virus.

\section{ROC analyses for selecting the optimal cutoff values for FIB-4 index and APRI}

In ROC analyses for HCC incidence at 5-year (almost equal to median follow-up period for the entire cohort), optimal cutoff point in FIB-4 index was 3.666 (AUROC $=0.768$; sensitivity, 68.0\%; and specificity, $80.47 \%)$, whereas that in APRI was 0.786 (AUROC $=0.629$; sensitivity, $84.0 \%$; and specificity, $43.2 \%$ ). We defined patients with FIB-4 index $\geq 3.666$ as high FIB-4 index group (H-FIB group, $n=83$ ) and patients with FIB-4 index $<3.666$ as low FIB-4 index group (L-FIB group, $\mathrm{n}=255$ ). Similarly, we defined patients with APRI $\geq 0.786$ as high APRI group (H-APRI group, $\mathrm{n}=188$ ) and patients with APRI $<0.786$ as low APRI group (L-APRI group, $n=150$ ). 


\section{Cumulative HCC incidence for all cases, $\mathrm{H}$ - and L- FIB groups and $\mathrm{H}$ - and L- APRI groups}

During the observation period, 33 patients $(9.8 \%)$ developed HCC. The median follow-up interval from the start of ETV therapy to the date of first confirmed HCC on imaging findings was 3.72 years. In patients with HCC incidence, FIB-4 index ranged from 0.981 to 11.682 (median, 3.900) and APRI ranged from 0.405 to 5.093 (median, 1.332). The 3-, 5and 7-year cumulative HCC incidence rates in all cases were $4.4 \%, 9.2 \%$ and $13.5 \%$, respectively. (Fig. 1A) In H- and L-FIB groups, the 3-, 5- and 7-year cumulative HCC incidence rates were $11.26 \%, 25.28 \%$ and $32.10 \%$, respectively, in the H-FIB group, and $2.09 \%, 3.99 \%$ and $7.48 \%$, respectively, in the L-FIB group $(P<0.0001)$. (Fig. 1B) In H- and L-APRI groups, the 3-, 5- and 7-year cumulative HCC incidence rates were $6.63 \%, 13.37 \%$ and $18.49 \%$, respectively, in the H-APRI group, and $1.45 \%, 3.71 \%$ and $6.85 \%$, respectively, in the L-APRI group $(P=0.0027)$. (Fig. 1C)

\section{Univariate and multivariate analyses of factors associated with HCC incidence}

Univariate analysis identified the following variables as significantly linked to HCC development for all cases: age $\geq 58$ years $(P<0.0001)$; presence of $\mathrm{LC}$ $(P<0.0001)$; serum albumin $\geq 3.9 \mathrm{~g} / \mathrm{dl}(P=0.0325)$; total bilirubin $\geq 1.1 \mathrm{mg} / \mathrm{dl}(P=0.0043)$; platelet count $\geq 14.1$ $\times 10^{4} / \mathrm{mm}^{3}(P<0.0001)$; APRI $\geq 0.786(P=0.0027)$; FIB-4 index $\quad \geq 3.666 \quad(P<0.0001)$; pretreatment alpha-fetoprotein (AFP) $\geq 13.2 \mathrm{ng} / \mathrm{ml}(P=0.0036)$; and pretreatment HBV-DNA $\geq 7.4$ log copies $/ \mathrm{ml}$ $(P=0.0098)$. (Table 2$)$ The odds ratios (ORs) and $95 \%$ confidence intervals (CIs) calculated using multivariate analysis for the nine factors with $P<0.05$ in univariate analysis are presented in table 2 . Age $\geq 58$ years $(P=0.0003)$, presence of LC $(P=0.0167)$, FIB-4 index $\geq 3.666(P=0.0374)$, pretreatment $\mathrm{AFP} \geq 13.2$ $\mathrm{ng} / \mathrm{ml}(P=0.0022)$ and pretreatment HBV-DNA $\geq 7.4$ $\log$ copies $/ \mathrm{ml}(P=0.0061)$ were found to be significant prognostic factors linked to the HCC development.
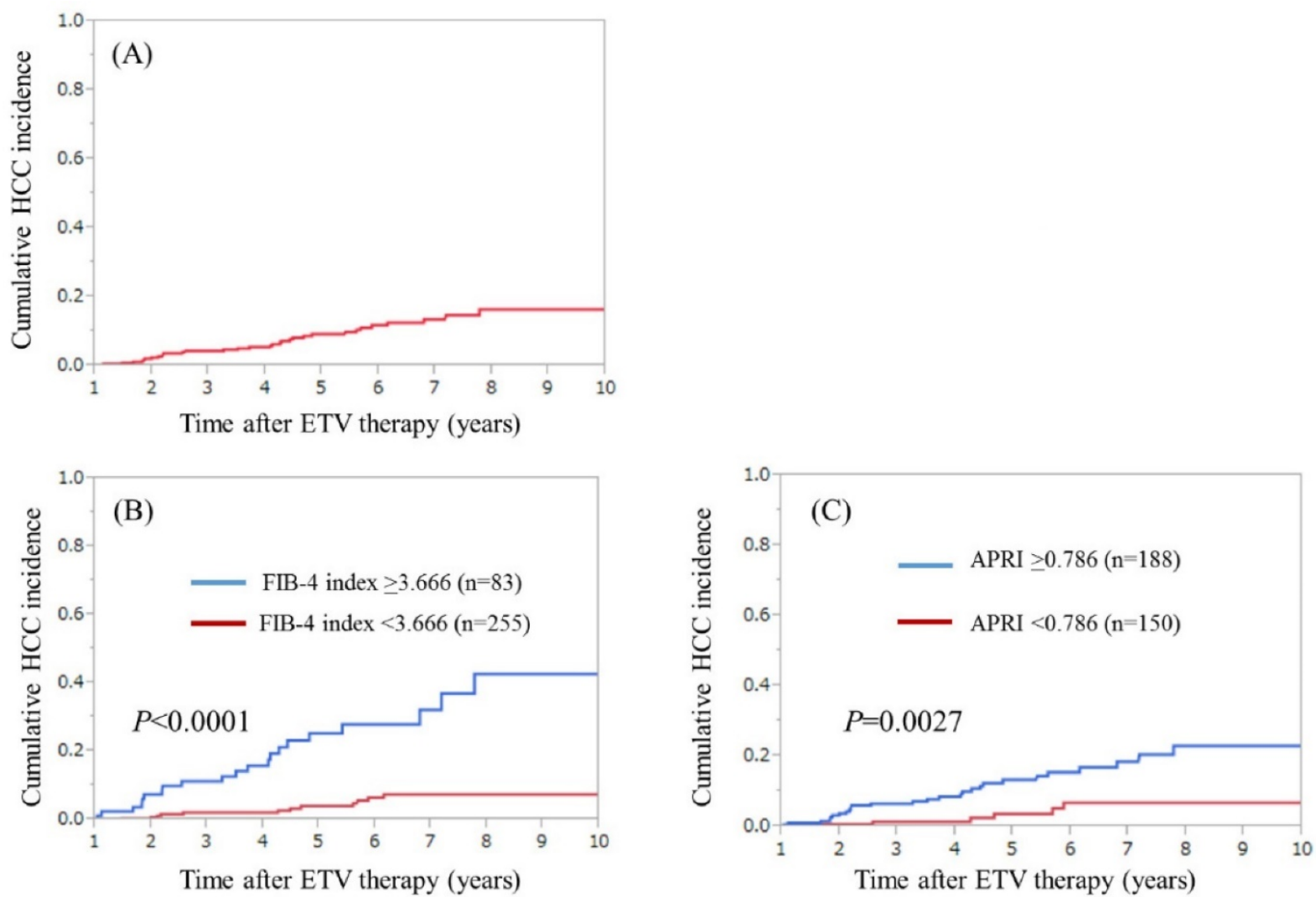

Figure 1. (A) Cumulative HCC incidence for all cases $(\mathrm{n}=338$ ). The 3-, 5- 7-year cumulative $\mathrm{HCC}$ incidence rates in all cases were $4.4 \%$, $9.2 \%$ and $13.5 \%$, respectively. (B) Cumulative HCC incidence in the high-FIB group (FIB-4 index $\geq 3.666, n=83$ ) and the low-FIB group (FIB-4 index $<3.666, n=255$ ). The 3-, 5- 7-year cumulative HCC incidence rates were $11.26 \%, 25.28 \%$ and $32.10 \%$, respectively, in the $\mathrm{H}$-FIB group, and $2.09 \%, 3.99 \%$ and $7.48 \%$, respectively, in the L-FIB group $(P<0.0001)$. (C) Cumulative HCC incidence in the $\mathrm{H}$ - APRI group (APRI $\geq 0.786, \mathrm{n}=188$ ) and the L-APRI group (APRI $<0.786, n=150$ ). The 3-, 5- 7-year cumulative HCC incidence rates were $6.63 \%, 13.37 \%$ and $18.49 \%$, respectively, in the $\mathrm{H}-\mathrm{APRI}$ group, and $1.45 \%, 3.71 \%$ and $6.85 \%$, respectively, in the $\mathrm{L}-\mathrm{APRI}$ group $(P=0.0027)$. 
Table 2. Univariate and multivariate analyses of factors contributing to HCC development.

\begin{tabular}{|c|c|c|c|c|}
\hline \multirow[t]{2}{*}{ Variables } & \multirow[t]{2}{*}{$\mathrm{n}$} & Univariate & \multicolumn{2}{|l|}{ Multivariate analysis } \\
\hline & & $P$ value & OR $(95 \% \mathrm{CI})$ & $P$ value \\
\hline Age $\geq 58$ years, yes / no & $118 / 220$ & $<0.0001$ & $4.348(1.952-10.477)$ & 0.0003 \\
\hline Gender, male / female & $215 / 123$ & 0.1732 & & \\
\hline Presence of LC, yes / no & $91 / 247$ & $<0.0001$ & $3.037(1.221-7.849)$ & 0.0167 \\
\hline AST $\geq 97 \mathrm{IU} / 1$, yes / no & $61 / 277$ & 0.1156 & & \\
\hline $\mathrm{ALT} \geq 59 \mathrm{IU} / 1$, yes / no & $160 / 178$ & 0.0511 & & \\
\hline Serum albumin $\geq 3.9 \mathrm{~g} / \mathrm{dl}$, yes $/$ no & 247 / 91 & 0.0325 & $0.658(0.281-1.506)$ & 0.3232 \\
\hline Total bilirubin $\geq 1.1 \mathrm{mg} / \mathrm{dl}$, yes / no & $86 / 252$ & 0.0043 & $1.056(0.492-2.302)$ & 0.8886 \\
\hline Platelets $\geq 14.1 \times 10^{4} / \mathrm{mm}^{3}$, yes $/$ no & $232 / 106$ & $<0.0001$ & $0.779(0.314-2.041)$ & 0.6018 \\
\hline APRI $\geq 0.786$, yes / no & $188 / 150$ & 0.0027 & $1.128(0.342-3.706)$ & 0.8386 \\
\hline FIB-4 index $\geq 3.666$, yes / no & 83 / 255 & $<0.0001$ & $2.946(1.063-9.223)$ & 0.0374 \\
\hline Pretreatment AFP $\geq 13.2 \mathrm{ng} / \mathrm{ml}$, yes / no & $75 / 263$ & 0.0036 & $3.312(1.556-6.996)$ & 0.0022 \\
\hline HBV-DNA $\geq 7.4 \log$ copies / ml, yes / no & $103 / 235$ & 0.0098 & $3.841(1.425-13.439)$ & 0.0061 \\
\hline HBe antigen positivity, yes / no & $163 / 175$ & 0.1209 & & \\
\hline
\end{tabular}

OR; hazard ratio, CI; confidence interval, LC; liver cirrhosis, AST; aspartate aminotransferase, ALT; alanine aminotransferase, APRI; AST to platelet ratio index, AFP. alpha-fetoprotein, HBV; hepatitis B virus

Table 3. Univariate and multivariate analyses of factors contributing to HCC development.

\begin{tabular}{|c|c|c|c|c|}
\hline \multirow[t]{2}{*}{ Variables } & \multirow[t]{2}{*}{$\mathrm{n}$} & Univariate & \multicolumn{2}{|l|}{ Multivariate analysis } \\
\hline & & $P$ value & OR $(95 \% \mathrm{CI})$ & $P$ value \\
\hline Gender, male / female & 215 / 123 & 0.1732 & & \\
\hline Presence of LC, yes / no & $91 / 247$ & $<0.0001$ & $2.603(1.089-6.476)$ & 0.0311 \\
\hline Serum albumin $\geq 3.9 \mathrm{~g} / \mathrm{dl}$, yes $/$ no & $247 / 91$ & 0.0325 & $0.824(0.362-1.832)$ & 0.6367 \\
\hline Total bilirubin $\geq 1.1 \mathrm{mg} / \mathrm{dl}$, yes / no & $86 / 252$ & 0.0043 & $1.150(0.536-2.421)$ & 0.7147 \\
\hline APRI $\geq 0.786$, yes / no & $188 / 150$ & 0.0027 & $1.037(0.309-3.460)$ & 0.9515 \\
\hline FIB-4 index $\geq 3.666$, yes / no & 83 / 255 & $<0.0001$ & $3.597(1.421-10.374)$ & 0.0062 \\
\hline Pretreatment AFP $\geq 13.2 \mathrm{ng} / \mathrm{ml}$, yes / no & 75 / 263 & 0.0036 & $2.221(1.074-4.527)$ & 0.0318 \\
\hline HBV-DNA $\geq 7.4 \log$ copies/ml, yes / no & $103 / 235$ & 0.0098 & $3.846(1.467-13.227)$ & 0.0045 \\
\hline HBe antigen positivity, yes / no & $163 / 175$ & 0.1209 & & \\
\hline
\end{tabular}

OR; hazard ratio, CI; confidence interval, LC; liver cirrhosis, AST; aspartate aminotransferase, ALT; alanine aminotransferase, APRI; AST to platelet ratio index, AFP; alpha-fetoprotein, HBV; hepatitis B virus.

\section{Univariate and multivariate analyses of factors associated with HCC incidence after excluding age, AST, ALT and platelet count from analysis}

Since FIB-4 index derived from age, AST, ALT and platelet count and APRI derived from AST and platelet count, we further performed univariate and multivariate analyses after excluding age, AST, ALT and platelet count from analysis.

In multivariate analysis, the following variables were associated with HCC incidence: presence of LC $(P=0.0311), \quad$ FIB-4 index $\geq 3.666 \quad(P=0.0062)$, pretreatment AFP $\geq 13.2 \mathrm{ng} / \mathrm{ml} \quad(P=0.0318)$ and pretreatment HBV-DNA $\geq 7.4$ log copies $/ \mathrm{ml}$ $(P=0.0045)$. The ORs and $95 \% \mathrm{CI}$ of these significant variables were presented in table 3 .

\section{Time-dependent ROC analyses for HCC development in all cases}

Results for time-dependent ROC analyses at 2-, 3-, 4-, 5-, 6- and 7-year of FIB-4 index and APRI in all cases were shown in fig. 2 and 3. All AUROCs of FIB-4 index in each time point were higher than those of APRI, suggesting that FIB-4 index had superior predictive ability for HCC incidence over APRI.

\section{Time-dependent ROC analyses for HCC development in patients with and without LC}

Results for time-dependent ROC analyses at 2-, 3-, 4-, 5-, 6- and 7-year of FIB-4 index and APRI in patients with LC $(n=91)$ were shown in fig. 4 A. All AUROCs of FIB-4 index in each time point were higher than those of APRI. Similarly, data for time-dependent ROC analyses at 3-, 4-, 5-, 6- and 7 -year of FIB-4 index and APRI in patients without LC $(n=247)$ were demonstrated in fig. 4B. None in patients without LC developed HCC within 2 years after the start of ETV therapy. All AUROCs of FIB-4 index in each time point were higher than those of APRI.

\section{Time-dependent ROC analyses for HCC development in patients with and without $\mathrm{HBe}$ antigen positivity}

Results for time-dependent ROC analyses at 2-, 3-, 4-, 5-, 6- and 7-year of FIB-4 index and APRI in patients with $\mathrm{HBe}$ antigen positivity $(n=163)$ were presented in fig. 4C. All AUROCs of FIB-4 index in each time point were higher than those of APRI. Likewise, data for time-dependent ROC analyses at 2-, 3-, 4-, 5-, 6- and 7-year of FIB-4 index and APRI in 
patients without $\mathrm{HBe}$ antigen positivity $(\mathrm{n}=175)$ were shown in fig. 4D. All AUROCs of FIB-4 index in each time point were higher than those of APRI.

\section{Time-dependent ROC analyses for HCC development in patients with baseline HBV-DNA $\geq 7.4$ log copies $/ \mathrm{ml}$ and baseline HBV-DNA $<7.4$ log copies $/ \mathrm{ml}$}

Results for time-dependent ROC analyses at 2-, $3-, 4-, 5-, 6-$ and 7-year of FIB-4 index and APRI in patients with HBV-DNA $\geq 7.4 \log$ copies/ml $(\mathrm{n}=103)$ were shown in fig. 5A. All AUROCs of FIB-4 index in each time point were higher than those of APRI. In the same way, data for time-dependent ROC analyses at 2-, 3-, 4-, 5-, 6- and 7-year of FIB-4 index and APRI in patients with HBV-DNA $<7.4$ log copies/ml $(\mathrm{n}=235)$ were presented in fig. 5B. All AUROCs of FIB-4 index in each time point were higher than those of APRI.

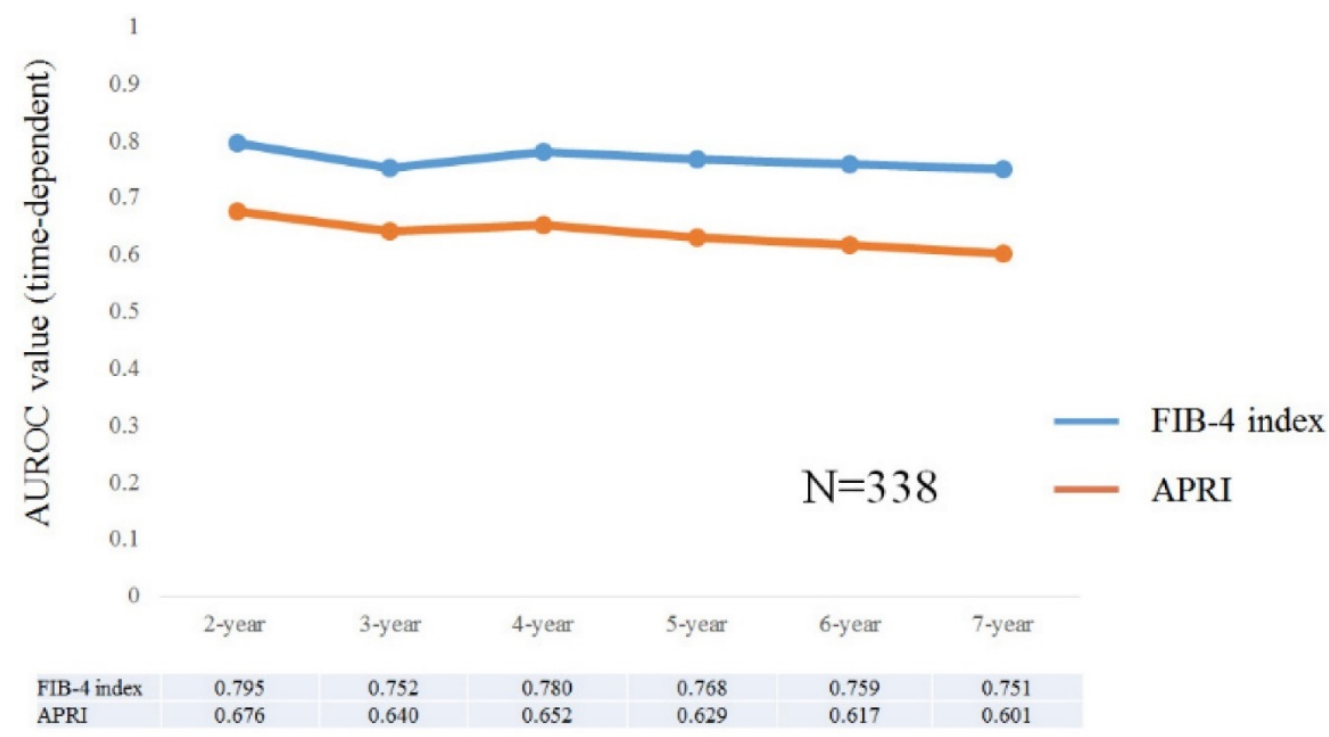

Figure 2. Data for time-dependent ROC analysis. Plots of annual AUROCs of FIB-4 index and APRI for HCC incidence after ETV therapy for all cases ( $\mathrm{n}=338$ ).

\section{FIB-4 index}

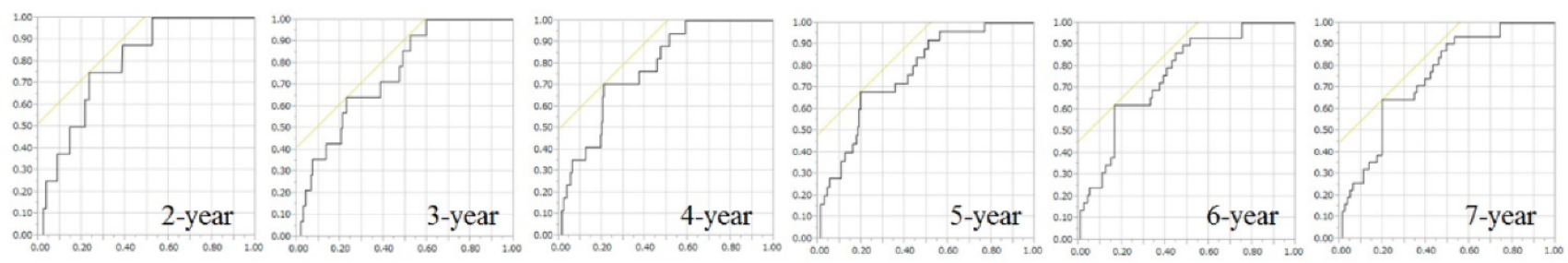

\section{APRI}
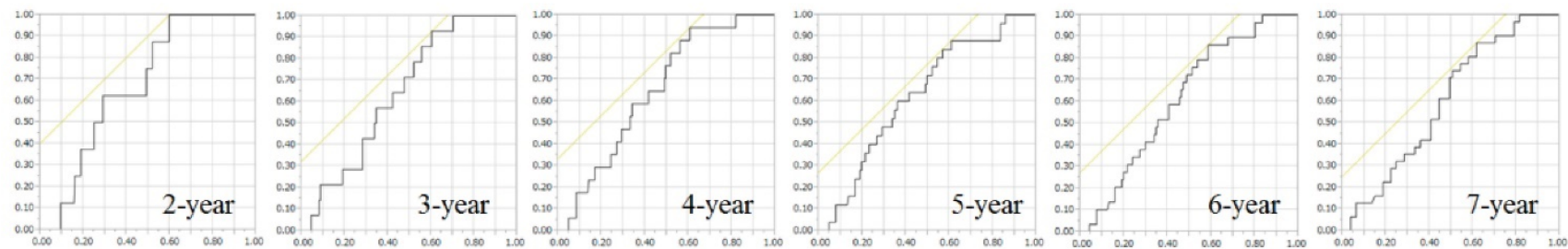

Figure 3. ROC curves for FIB-4 index and APRI at 2-, 3-, 4-, 5-, 6- and 7-year time points. Vertical axis indicates sensitivity and horizontal axis indicates 1-specificity. 

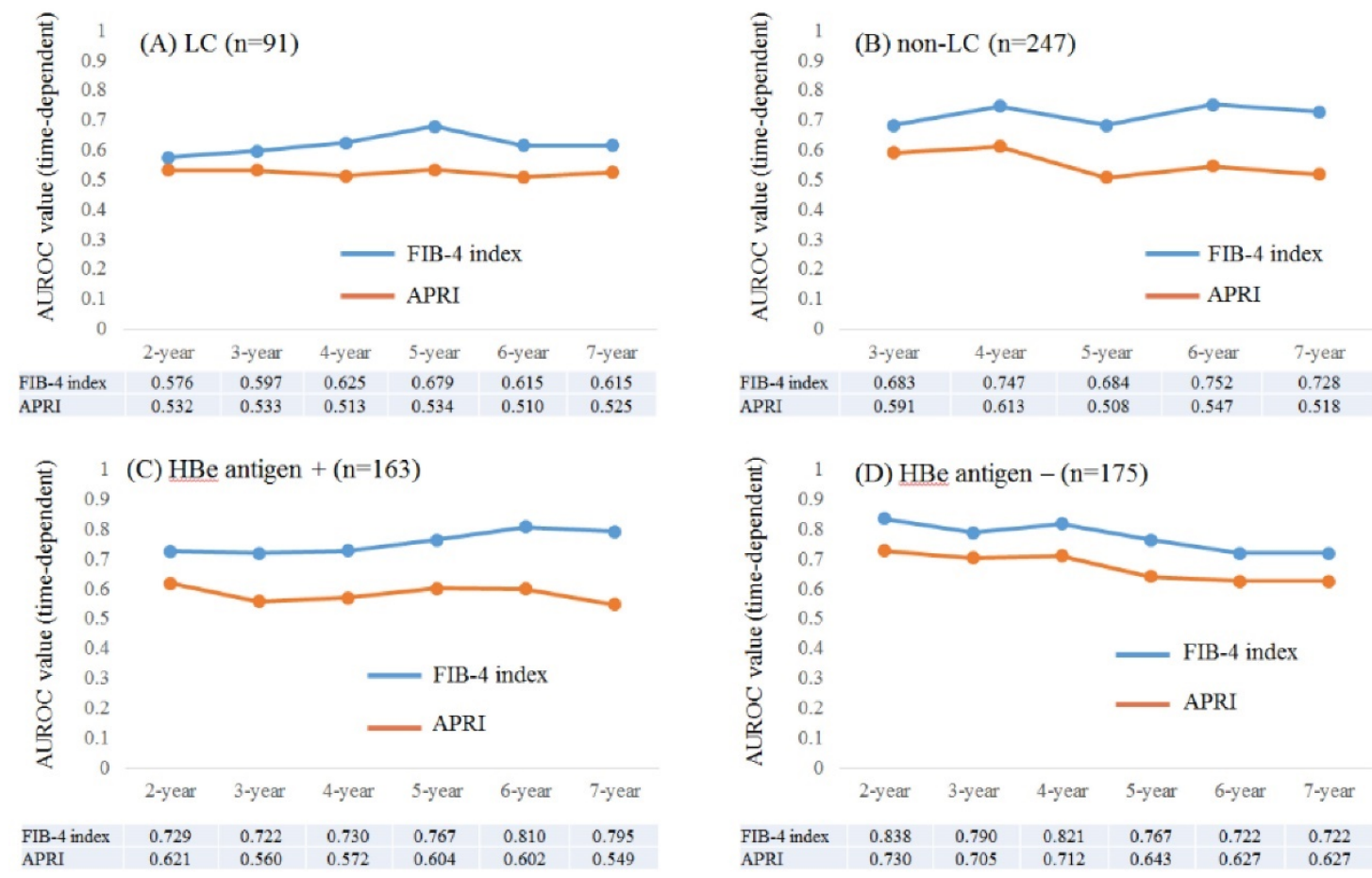

Figure 4. Subgroup analyses. Plots of annual AUROCs of FIB-4 index and APRI for HCC incidence after ETV therapy for patients with LC ( $n=91)(A)$ and without LC $(n=247)(B)$. No patient in non-LC developed HCC within 2 years after the start of ETV therapy. Plots of annual AUROCs of FIB-4 index and APRI for HCC incidence after ETV therapy for patients with $\mathrm{HBe}$ antigen positivity $(n=163)(C)$ and without HBe antigen positivity $(n=175)(D)$.
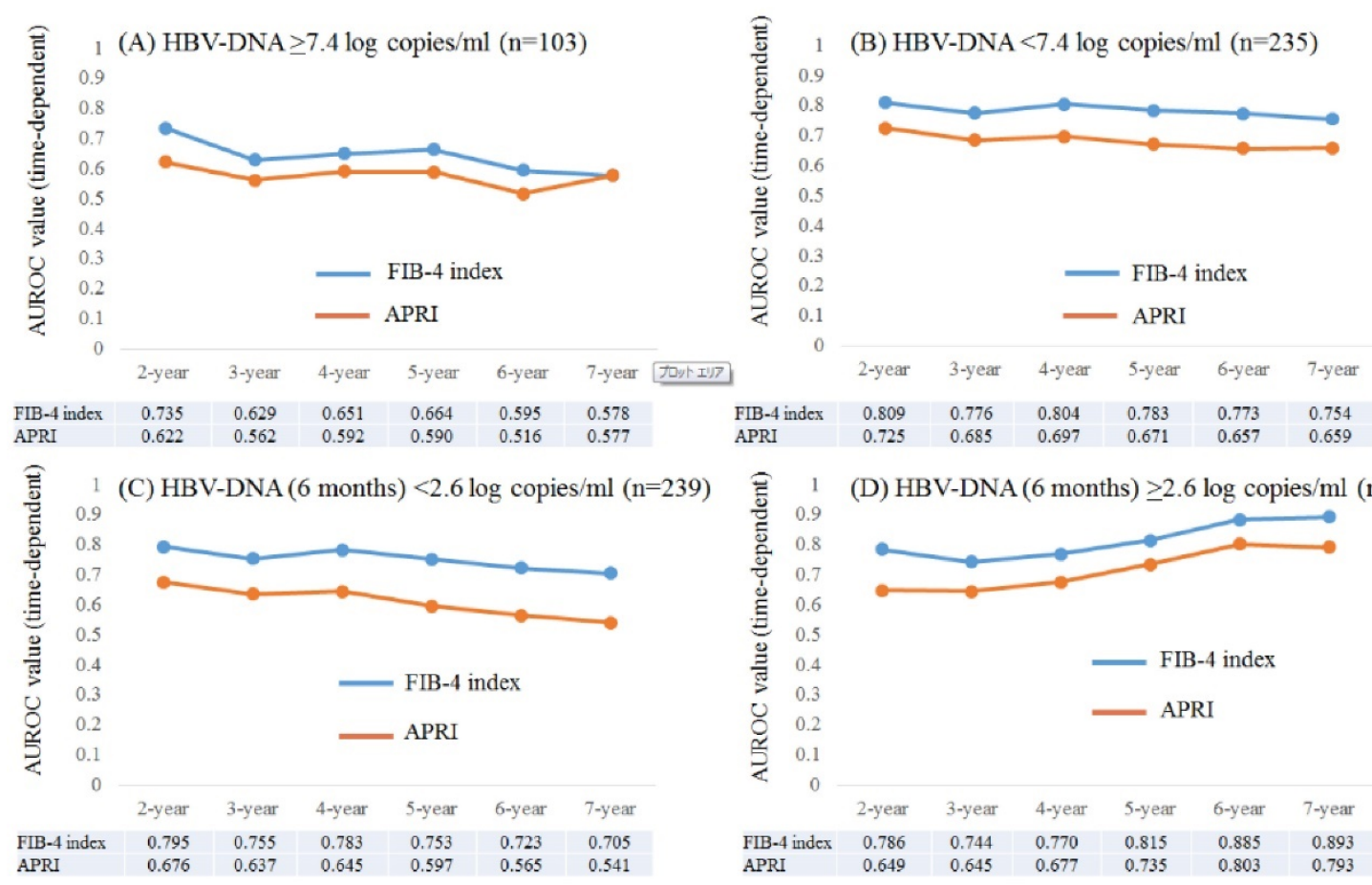

Figure 5. Subgroup analyses. Plots of annual AUROCs of FIB-4 index and APRI for HCC incidence after ETV therapy for patients with HBV-DNA $>7.4$ log copies/ml at baseline $(n=103)(A)$ and HBV-DNA $<7.4 \log$ copies $/ \mathrm{ml}$ at baseline $(n=235)$ (B). Plots of annual AUROCs of FIB-4 index and APRI for HCC incidence after ETV therapy for patients with HBV-DNA $<2.6$ log copies/ml at 6 months $(n=239)$ (C) and HBV-DNA $\geq 2.6$ log copies/ml at 6 months $(n=99)(D)$. 


\section{Time-dependent ROC analyses for HCC development in patients with HBV-DNA (6 months) $<2.6 \mathrm{log}$ copies/ml and HBV-DNA (6 months) $\geq \mathbf{2 . 6}$ log copies $/ \mathrm{ml}$}

Results for time-dependent ROC analyses at 2-, 3-, 4-, 5-, 6- and 7-year of FIB-4 index and APRI in patients with HBV-DNA (6 months) $<2.6 \quad$ log copies $/ \mathrm{ml} \quad(\mathrm{n}=239)$ were shown in fig. 5C. All AUROCs of FIB-4 index in each time point were higher than those of APRI. Likewise, data for time-dependent ROC analyses at 2-, 3-, 4-, 5-, 6- and 7-year of FIB-4 index and APRI in patients with HBV-DNA (6 months) $\geq 2.6 \log$ copies/ $\mathrm{ml}(\mathrm{n}=99)$ were demonstrated in fig. 5D. All AUROCs of FIB-4 index in each time point were higher than those of APRI.

\section{Discussion}

To the best of our knowledge, there have been no reports regarding the comparison of effects of FIB-4 index and APRI on HCC incidence in CHB patients undergoing ETV therapy. Although FIB-4 index and APRI can be easily calculated by routine laboratory blood tests and are well established non-invasive liver fibrosis markers, whether these markers can be helpful for predicting HCC incidence for CHB patients treated with ETV remains an unsolved problem. [25, 29, 30, 35] Additionally, despite the fact that most disease clinical outcomes are time-dependent, no previous reports have performed time-dependent ROC analysis to fully assess the clinical implication of non-invasive liver fibrosis markers in terms of HCC incidence for CHB patients undergoing ETV therapy. We therefore performed this investigation. A notable strength in this study is the homogenous Japanese CHB patient cohort, which eliminated the confounding effects of patient ethnicity.

In our data, FIB-4 index was an independent predictor for HCC incidence before and after excluding age, AST, ALT and platelet count from analysis, while APRI was not. Furthermore, in the time-dependent ROC analysis, FIB-4 index had higher AUROCs than APRI at all time points for all cases and for all subgroup analyses. These results indicate that FIB-4 index was superior to APRI for predictability of HCC incidence in $\mathrm{CHB}$ patients undergoing ETV therapy regardless of $\mathrm{HBV}$ status, LC status at baseline or treatment response. In the clinical settings, patients with higher FIB-4 index should be closely monitored for HCC incidence. One potential explanation for current results is that FIB-4 index includes age, whereas APRI does not, which is the major difference in these two liver fibrosis markers. Indeed, in the multivariate analysis before excluding age, AST, ALT and platelet count, older age was an independent predictor linked to HCC development. In addition, the prevalence of LC in the H-FIB group $(66.3 \%, 55 / 83)$ was significantly higher than that in the H-APRI group $(41.0 \%, 77 / 18)(P=0.0001)$, which is probably the most plausible explanation for better predictability for HCC incidence of FIB-4 index than ARPI, because presence of LC is a well-known predictor for HCC incidence in $\mathrm{CHB}$ patients. $[7,10$, 12-17]

Arends, et al. reported that the 5-year cumulative $\mathrm{HCC}$ incidence rate in $\mathrm{CHB}$ patients undergoing ETV therapy with various racial backgrounds was $2.1 \%$ for non-LC and $10.9 \%$ for LC patients $(P<0.001)$, whereas in our data, the 5-year cumulative HCC incidence rate was $4.5 \%$ for non-LC and $21.5 \%$ for LC patients $(P<0.0001)$, which were higher than Arends, et al. data. [36] Different racial backgrounds or HBV-DNA genotypes may explain these discrepancies. [37] Disease progression for HCC are more frequently encountered with $\mathrm{CHB}$ patients with genotypes $\mathrm{C}$ and $\mathrm{D}$ than those with the other genotypes and genotype $\mathrm{C}$ is in the majority in Japanese CHB patients. [18, 37, 38]

The correlation between HBV-DNA level at baseline and future risk for HCC is one of recent major progresses in our understanding for the natural clinical course of CHB infection. [10] In our results, higher HBV-DNA level at baseline was significantly linked to HCC incidence, which are in line with previous reports. [10] On the other hand, Tada, et al. demonstrated in their large cohort that $\mathrm{HB}$ core related $\mathrm{Ag}$ (HBcrAg) was superior to HBV-DNA for predicting HCC incidence. [39] The comparison of FIB-4 index and HBcrAg in terms of prognostic impact for HCC incidence in CHB patients could be a point of focus in the future.

Notably, in patients with HBV-DNA at 6 months $\geq 2.6 \log$ copies $/ \mathrm{ml}$, AUROC in time-dependent analysis at 5-, 6- and 7- years of FIB-4 index were more than 0.8 . These results indicated that in patients with poor response to antiviral therapy in the early stage, the effect of FIB-4 index for predicting carcinogenesis is prominent in the later stage of the follow-up period. Especially in patients with poor early response to ETV and higher FIB-4 index at baseline, closely regular HCC surveillance will be required.

HBV-related HCC may develop in non-LC patients. [40] In our analysis, out of 33 patients with HCC incidence, 11 patients $(33.3 \%)$ had non-LC status. The median time interval for the date of ETV therapy to the date of first confirmed HCC incidence in these patients was 4.26 years. These results support the notion that HBV itself plays a direct role in the liver transformation by triggering carcinogenic 
pathways. [19] It should be noted for clinicians that HBV contributes to development of HCC through these direct mechanisms and liver fibrosis related indirect mechanisms, although this is beyond the aim of our study.

There are limitations to our study. Firstly, as our study findings were limited by the retrospective nature of our study design, we should interpret our study data with caution. Secondly, as our study subjects were limited for patients initially treated with ETV, whether our results could be applied to CHB patients receiving other NAs needs further research. Thirdly, as the study subjects were all recruited from Japanese patients, generalizability of this study findings to patients with other ethnicities remains to be evaluated. Finally, we did not have sufficient numbers of HCC incidence for analysis, potentially leading to bias. Thus, future well designed larger studies will be needed to consolidate our results. However, despite the aforementioned study limitations, our current results presented that FIB-4 index was superior to APRI for predicting HCC incidence in $\mathrm{CHB}$ patients undergoing ETV therapy. In conclusion, FIB-4 index rather than APRI can be a useful predictor associated with HCC development for CHB patients treated with ETV. An emphasis is warranted for the easy and prompt clinical utility of FIB-4 index for predicting HCC incidence as well as the degree of liver fibrosis.

\section{Abbreviations}

HCC: hepatocellular carcinoma; CHB: Chronic hepatitis B; HBV: hepatitis B virus; HBs: hepatitis B surface ALT: alanine aminotransferase; LC: liver cirrhosis; AASLD: The American Association for Study of Liver Disease; ETV: entecavir; NAs: nucleoside analogues; AST: aspartate aminotransferase; APRI: AST to platelet ration index; ROC: receiver operating characteristic curve; AUROCs: area under the ROCs AFP: alpha-fetoprotein; OR: odds ratio; CI: confidence interval; HBcrAg: HB core related antigen.

\section{Acknowledgement}

The authors would like to thank Nozomi Kanazawa (Hyogo college of medicine), Yoko Matsushita (Hyogo college of medicine), Sayaka Fujii (Hyogo college of medicine) and Haruko Takada (Osaka Red Cross hospital) for data acquisition.

\section{Author contribution}

Hiroki Nishikawa, Norihiro Nishijima and Hirayuki Enomoto analysed data and wrote the paper and other remaining authors collected data.

\section{Conflict of Interest}

In the present study, the authors declare that they have no conflicts of interest.

\section{References}

[1] El-Serag HB. Hepatocellular carcinoma. N Engl J Med. 2011; 365: 1118-1127.

[2] Sherman M. Epidemiology of hepatocellular carcinoma. Oncology. 2010; 78(Suppl 1): 7-10.

[3] Jemal A, Bray F, Center MM, Ferlay J, Ward E, Forman D. Global cancer statistics. CA Cancer J Clin. 2011; 61(2): 69-90.

[4] McMahon BJ. The natural history of chronic hepatitis B virus infection. Hepatology. 2009; 49(5 Suppl): S45-55.

[5] Singal AG, El-Serag HB. Hepatocellular Carcinoma From Epidemiology to Prevention: Translating Knowledge into Practice. Clin Gastroenterol Hepatol. 2015; 13(12): 2140-2151.

[6] Schweitzer A, Horn J, Mikolajczyk RT, Krause G, Ott JJ. Estimations of worldwide prevalence of chronic hepatitis B virus infection: a systematic review of data published between 1965 and 2013. Lancet. 2015; 386(10003): 1546-1555.

[7] Lin CL, Yang HC, Kao JH. Hepatitis B virus: new therapeutic perspectives. Liver Int. 2016; 36 Suppl 1: 85-92.

[8] Sundaram V, Kowdley K. Management of chronic hepatitis B infection. BMJ. 2015 Oct 21; 351: h4263.

[9] Kwon H, Lok AS. Hepatitis B therapy. Nat Rev Gastroenterol Hepatol. 2011; 8(5): 275-284.

[10] Chen CJ, Yang HI, Su J, Jen CL, You SL, Lu SN, et al. Risk of hepatocellular carcinoma across a biological gradient of serum hepatitis B virus DNA level. JAMA 2006; 295: 65-73.

[11] Koh C, Zhao X, Samala N, Sakiani S, Liang TJ, Talwalkar JA. AASLD clinical practice guidelines: a critical review of scientific evidence and evolving recommendations. Hepatology. 2013; 58(6): 2142-2152.

[12] Hosaka T, Suzuki F, Kobayashi M, Seko Y, Kawamura Y, Sezaki H, et al. Long-term entecavir treatment reduces hepatocellular carcinoma incidence in patients with hepatitis B virus infection. Hepatology. 2013; 58(1): 98-107.

[13] Yamada R, Hiramatsu N, Oze T, Morishita N, Harada N, Yakushijin T, et al; Osaka Liver Forum. Impact of alpha-fetoprotein on hepatocellular carcinoma development during entecavir treatment of chronic hepatitis B virus infection. J Gastroenterol. 2015; 50(7): 785-794.

[14] Wong VW, Chan SL, Mo F, Chan TC, Loong HH, Wong GL, et al. Clinical scoring system to predict hepatocellular carcinoma in chronic hepatitis B carriers. J Clin Oncol. 2010; 28: 1660-1665.

[15] Yang HI, Sherman M, Su J, Chen PJ, Liaw YF, Iloeje UH, et al. Nomograms for risk of hepatocellular carcinoma in patients with chronic hepatitis B virus infection. J Clin Oncol. 2010; 28: 2437-2444

[16] Yang HI, Yuen MF, Chan HL, Han KH, Chen PJ, Kim DY, et al, Risk estimation for hepatocellular carcinoma in chronic hepatitis B (REACH-B): development and validation of a predictive score. Lancet Oncol. 2011; 12: 568-574.

[17] Yuen MF, Tanaka Y, Fong DY, Fung J, Wong DK, Yuen JC, et al. Independent risk factors and predictive score for the development of hepatocellular carcinoma in chronic hepatitis B. J Hepatol. 2009; 50: 80-88.

[18] Orito E, Mizokami M. Hepatitis B virus genotypes and hepatocellular carcinoma in Japan. Intervirology. 2003; 46(6): 408-412.

[19] Ali A, Abdel-Hafiz H, Suhail M, Al-Mars A, Zakaria MK, Fatima K, et al. Hepatitis B virus, $\mathrm{HBx}$ mutants and their role in hepatocellular carcinoma. World J Gastroenterol. 2014; 20(30): 10238-10248.

[20] Kim WR, Loomba R, Berg T, Aguilar Schall RE, Yee LJ, Dinh PV, et al. Impact of long-term tenofovir disoproxil fumarate on incidence of hepatocellular carcinoma in patients with chronic hepatitis B. Cancer. 2015; 121(20): 3631-3638.

[21] Lok AS, McMahon BJ, Brown RS Jr, Wong JB, Ahmed AT, Farah W, et al. Antiviral therapy for chronic hepatitis B viral infection in adults: A systematic review and meta-analysis. Hepatology. 2016; 63(1): 284-306.

[22] Wang YJ, Yang L, Zuo JP. Recent developments in antivirals against hepatitis B virus. Virus Res. 2016; 213: 205-213.

[23] Ono A, Suzuki F, Kawamura Y, Sezaki H, Hosaka T, Akuta N, et al. Long-term continuous entecavir therapy in nucleos(t)ide-naïve chronic hepatitis $B$ patients. J Hepatol. 2012; 57(3): 508-514.

[24] Wong GL, Chan HL, Mak CW, Lee SK, Ip ZM, Lam AT, et al. Entecavir treatment reduces hepatic events and deaths in chronic hepatitis B patients with liver cirrhosis. Hepatology. 2013; 58(5): 1537-1547.

[25] Xiao G, Yang J, Yan L. Comparison of diagnostic accuracy of aspartate aminotransferase to platelet ratio index and fibrosis-4 index for detecting liver fibrosis in adult patients with chronic hepatitis B virus infection: a systemic review and meta-analysis. Hepatology. 2015; 61(1): 292-302.

[26] Li Y, Chen Y, Zhao Y. The diagnostic value of the FIB-4 index for staging hepatitis B-related fibrosis: a meta-analysis. PLoS One. 2014; 9(8): e105728.

[27] Suh B, Park S, Shin DW, Yun JM, Yang HK, Yu SJ, et al. High liver fibrosis index FIB-4 is highly predictive of hepatocellular carcinoma in chronic hepatitis B carriers. Hepatology. 2015; 61(4): 1261-1268.

[28] Hann HW, Wan S, Lai Y, Hann RS, Myers RE, Patel F, et al. Aspartate aminotransferase to platelet ratio index as a prospective predictor of 
hepatocellular carcinoma risk in patients with chronic hepatitis B virus infection. J Gastroenterol Hepatol. 2015; 30(1): 131-138.

[29] Lin ZH, Xin YN, Dong QJ, Wang Q, Jiang XJ, Zhan SH, et al. Performance of the aspartate aminotransferase-to-platelet ratio index for the staging of hepatitis C-related fibrosis: an updated meta-analysis. Hepatology. 2011; 53(3): 726-736.

[30] Vallet-Pichard A, Mallet V, Nalpas B, Verkarre V, Nalpas A, Dhalluin-Venier $\mathrm{V}$, et al. FIB-4: an inexpensive and accurate marker of fibrosis in HCV infection. comparison with liver biopsy and fibrotest. Hepatology. 2007; 46(1): 32-36.

[31] Kaneko S, Furuse J, Kudo M, Ikeda K, Honda M, Nakamoto Y, et al. Guideline on the use of new anticancer drugs for the treatment of Hepatocellular Carcinoma 2010 update. Hepatol Res. 2012; 42(6): 523-542.

[32] Yamakado K, Kudo M. Treatment strategies of intermediate-stage hepatocellular carcinomas in Japan (Barcelona Clinic Liver Cancer stage B). Oncology. 2014; 87 Suppl 1: 78-81.

[33] Kudo M, Izumi N, Kokudo N, Matsui O, Sakamoto M, Nakashima O, et al: HCC Expert Panel of Japan Society of Hepatology. Management of hepatocellular carcinoma in Japan: Consensus-Based Clinical Practice Guidelines proposed by the Japan Society of Hepatology (JSH) 2010 updated version. Dig Dis. 2011; 29(3): 339-364.

[34] Heagerty PJ, Zheng Y. Survival model predictive accuracy and ROC curves. Biometrics. 2005; 61(1): 92-105.

[35] Shaheen AA, Myers RP. Diagnostic accuracy of the aspartate aminotransferase-to-platelet ratio index for the prediction of hepatitis C-related fibrosis: a systematic review. Hepatology. 2007; 46(3): 912-921.

[36] Arends P, Sonneveld MJ, Zoutendijk R, Carey I, Brown A, Fasano M, et al; VIRGIL Surveillance Study Group. Entecavir treatment does not eliminate the risk of hepatocellular carcinoma in chronic hepatitis B: limited role for risk scores in Caucasians. Gut. 2015; 64(8): 1289-1295.

[37] Sunbul M. Hepatitis B virus genotypes: global distribution and clinical importance. World J Gastroenterol. 2014; 20(18): 5427-5434.

[38] Omata M, Lesmana LA, Tateishi R, Chen PJ, Lin SM, Yoshida H, et al. Asian Pacific Association for the Study of the Liver consensus recommendations on hepatocellular carcinoma. Hepatol Int. 2010; 4(2): 439-474.

[39] Tada T, Kumada T, Toyoda H, Kiriyama S, Tanikawa M, Hisanaga Y, et al. HBcrAg predicts hepatocellular carcinoma development: An analysis using time-dependent receiver operating characteristics. J Hepatol. 2016 Mar 28. E-pub ahead of print.

[40] Levrero M, Zucman-Rossi J. Mechanisms of HBV-induced hepatocellular carcinoma. J Hepatol. 2016; 64(1 Suppl): S84-S101. 